\title{
Cumulative use of therapeutic bladder anticholinergics and the risk of dementia in patients with lower urinary tract symptoms: a nationwide 12-year cohort study
}

Yi-Chi Wang ${ }^{1}$, Yung-Liang Chen², Chun-Che Huang ${ }^{3}$, Chung-Han Ho ${ }^{4,5}$, Yu-Tung Huang ${ }^{6}$, Ming-Ping Wu, Ming-Jung Ou', Chiu-Hsien Yang ${ }^{9}$ and Ping-Jen Chen ${ }^{9,10,11,12^{*}}$ (B)

\begin{abstract}
Background: Studies have shown an association between lower urinary tract symptoms (LUTS) and an increased risk of dementia. Whether anticholinergic use contributes to the development of dementia in patients with LUTS remains unknown, especially in Asian populations. This study aims to investigate the association between anticholinergic use and dementia in patients with LUTS.

Methods: This study included patients aged 50 years and over with newly diagnosed LUTS (January 2001 to December 2005), divided into four groups according to their cumulative defined daily doses (cDDDs) of anticholinergics: < 28 CDDDs, 28-84 CDDDs, 85-336 cDDDs, $\geq 337$ cDDDs. Patients were followed up until dementia developed or until the end of 2012.

Results: We recruited a total of 16,412 patients. The incidence of dementia was $10 \%$ in the < 28 cDDD group, $8.9 \%$ in the $28-84$ cDDD group, $11.5 \%$ in the $85-336$ cDDD group, and $14.4 \%$ in the $\geq 337$ cDDD group $(p=.005)$. In a Cox proportional hazards analysis, the adjusted hazard ratio of dementia was $1.15(95 \% \mathrm{Cl}=0.97-1.37)$ in the $85-$ 336 cDDD group, and $1.40(95 \% \mathrm{Cl}=1.12-1.75)$ in the $\geq 337$ cDDD group after adjusting for covariates.

Conclusions: Our study indicates that higher cumulative anticholinergic exposure is associated with an increase in the risk of incident dementia in patients with LUTS aged 50 years of age and over. Either using one anticholinergic agent or switching anticholinergic agents cumulatively increases this risk. Therapeutic risks and benefits of using anticholinergics in LUTS treatment should be clinically reviewed and weighed.
\end{abstract}

Keywords: Anticholinergics, Cohort study, Cumulative defined daily doses, Dementia, Lower urinary tract symptoms

\section{Background}

Lower urinary tract symptoms (LUTS) is a general term for symptoms related to urinary storage and/ or voiding disturbances [1], including frequency, urgency, nocturia, and incontinence. LUTS is a common medical issue in older people [2], and its prevalence and severity have been shown to increase even into the tenth decade of life [3]. LUTS have also been proven to interfere with

\footnotetext{
* Correspondence: pingjen.chen@gmail.com

${ }^{9}$ Department of Family Medicine, Kaohsiung Medical University Hospital, Kaohsiung Medical University, Kaohsiung City 807, Taiwan

${ }^{10}$ School of Medicine, College of Medicine, Kaohsiung Medical University, Kaohsiung City 807, Taiwan

Full list of author information is available at the end of the article
}

activities of daily living [2] and are associated with poorer quality of life [4]. The severity of LUTS are a risk factor for sleep disturbance [5], and these have been strongly associated with mental illness, such as depression and anxiety $[6,7]$. LUTS have a significant impact on healthcare costs [8], social welfare, and the healthcare system $[9,10]$.

Dementia is characteristically a disease of older people [11]. Worldwide, 48 million people currently live with dementia; due to the aging population, its prevalence is expected to triple by 2050 [12]. Dementia not only causes disability and death, but also increases the risk of requiring nursing home care [13] and contributes to 
burden on caregivers [14]. Despite an increasing global disease prevalence, effective therapy to treat dementia is still lacking [15]. To date, many potentially modifiable risk factors for dementia prevention have been proposed, including lifestyle management, vascular risk factor control, and nutritional support [16]; however, the overall evidence is not strong.

In our previous study, we found that LUTS were associated with an increased risk of dementia [17]. However, whether LUTS are a direct risk factor for dementia or whether there are confounding factors remains unknown. Voiding requires a complex mechanism concomitantly regulated by the brain and the urinary system. Evidence suggests that small vessel disease of the brain affects white matter, and white matter disease can cause LUTS, especially in older people [18].

Treatments for LUTS include behavioral therapy, medication, and surgery. Alpha adrenergic-receptor antagonists, 5-alpha reductase inhibitors, anticholinergics, beta 3 -adrenoceptor agonists, and phosphodiesterase 5 inhibitors are commonly used medications [19]. Anticholinergics play an important role in treating LUTS by blocking cholinergic muscarinic receptors in the bladder and decreasing involuntary detrusor contractions [20]. However, compelling evidence suggests that anticholinergics are also associated with an increased risk of brain atrophy, dysfunction [21], and dementia [22].

Whether bladder anticholinergic use contributes to increased risk of incident dementia in patients with LUTS is still unclear. Therefore, we used Taiwan's National Health Insurance Research Database (NHIRD) to conduct a cohort study to investigate our hypothesis that cumulative use of anticholinergics is associated with a higher risk of incident dementia.

\section{Methods \\ Data source}

This retrospective, population-based cohort study used data from the Longitudinal Health Insurance Database (LHID), from which 1 million beneficiaries were randomly selected from the NHIRD in Taiwan. There were no differences in age, sex, or average insured payroll-related premiums between the LHID sample and all NHIRD enrollees. Data on outpatient visits, hospital admissions, prescriptions, disease status, and demographics were retrieved from the LHID database. To protect confidentiality all patient and medical institution identification numbers were encrypted and maintained by the National Health Research Institutes of Taiwan before the data were released. The International Classification of Disease, 9th revision, Clinical Modification (ICD-9-CM) coding system was used to classify diagnoses in the LHID. The study was approved by the Institutional Review Board of Chi Mei Medical Center (IRB No. 10708-
E01), and the requirement for informed consent was waived.

\section{Study population}

A total of 19,273 patients with at least three outpatient visits or one inpatient admission with a principal diagnosis of LUTS (ICD-9-CM codes 596.51, 600.x, 625.6, 788.2, 788.31-788.33, 788.35, 788.36, 788.4 and 788.6) were identified from January 2001 to December 2005 to validate the accuracy of the diagnoses. We excluded patients aged younger than 50 years $(n=562)$, those who had been diagnosed with LUTS before the end of 2000 $(n=1955)$, and those diagnosed with dementia before the first diagnosis of LUTS $(n=344)$. The date of initial LUTS diagnosis was chosen as the index date. Patients with LUTS who received prescriptions for anticholinergics after the index date were divided into four groups according to their use of anticholinergics. A flow diagram of the sample selection is shown in Fig. 1 .

\section{Outcome}

The primary outcome was the occurrence of dementia, defined as a patient who had at least three outpatient visits or one inpatient admission for a principal diagnosis of dementia (ICD-9-CM 290.0-290.4 and 331.0) after the first diagnosis of LUTS. Patients with newly diagnosed dementia within the first year after the index date were excluded. Patients were followed up until development of dementia or until the end of 2012. All the patients were identified in the LHID database to ensure diagnostic accuracy.

\section{Anticholinergic drug exposure}

The primary independent variable of interest was the use of anticholinergics prescribed for patients with LUTS. We lack a gold standard measurement of anticholinergic effect of individual drugs on the human brain. The Anticholinergic Cognitive Burden (ACB) scale was developed in 2008 and updated in 2012. Drugs with higher ACB score [23] are associated with increased risk of cognitive function decline. Drugs with an ACB score of 1 (possibly anticholinergic) have antagonist activity at muscarinic receptors from in vitro data. Drugs with an ACB score of 2 (definitely anticholinergic) have clinical anticholinergic effect identified from literature reviews, prescriber's information or expert opinion. Drugs with an ACB score of 3 (definitely anticholinergic) not only have clinical anticholinergic effect, but are also associated with delirium from literature reviews, prescriber's information or expert opinion [23].

In our study, anticholinergic prescriptions were determined by using the Anatomical Therapeutic Chemical system of medications for Flavoxate (G04BD02), Oxybutynin (G04BD04), Propiverine (G04BD06), Tolterodine 


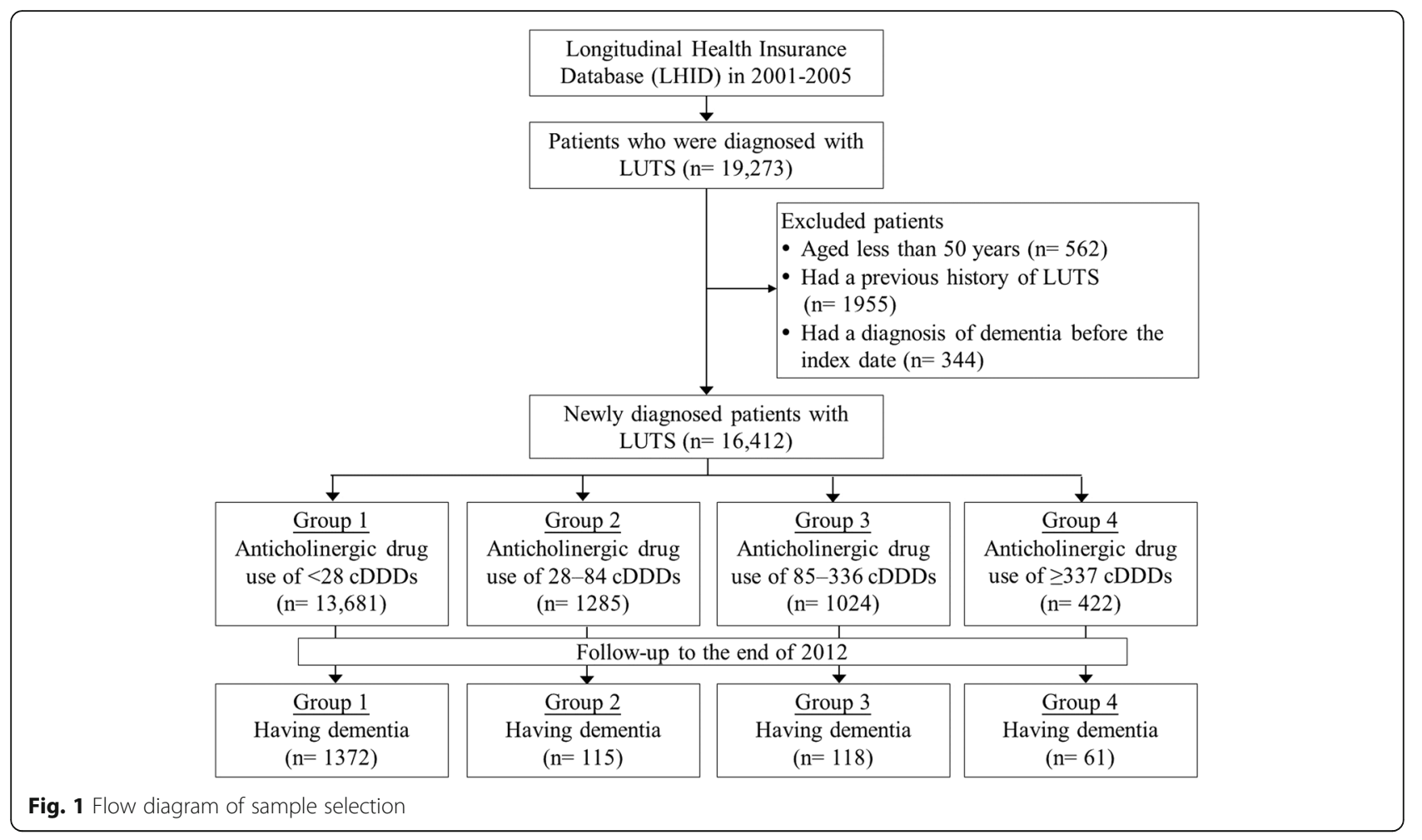

(G04BD07), Solifenacin (G04BD08), Trospium (G04BD09) from the LHID claims data. All the above drugs were available in Taiwan in treating LUTS and were classified into the ACB score group 3.

The defined daily doses (DDDs) were those recommended by the Collaborating Center for Drug Statistics Methodology of the World Health Organization (WHO) and reflected the assumed average maintenance dose per day for a drug used for its main indication in adults [24]. We used the formula to quantify the use of anticholinergics: (total amount of drug)/(amount of drug in a DDD) = number of DDDs [24]. To measure their risk of increasing dementia, cumulative DDDs (cDDDs) indicating the total exposed dosage of all anticholinergics in ACB score group 3 were estimated as the sum of dispensed DDDs of anticholinergics. We stratified the use of anticholinergics into four levels: < 28, 28-84, 85-336, and $\geq 337$ cDDDs.

\section{Covariates}

Covariates were selected as follows: patient's age at LUTS diagnosis, sex, catastrophic illness certificate, and comorbidities. Age was categorized into five groups: 50$59,60-69,70-79$, and $\geq 80$ years. We used the LHID registry for catastrophic illness certification to identify patients with catastrophic illness, including malignancies and other noncancerous major diseases, such as renal failure, chronic mental disorders, and autoimmune diseases, which were defined by the Bureau of National Health Insurance (NHI). Under Taiwan's NHI scheme, a certificate of catastrophic illness waives copayment by a patient for medical care, including for outpatient visits and inpatient admissions. Numbers of outpatient visits were defined as the average of annual outpatient visits per person after the index date. Comorbidities were determined by using ICD-9-CM codes as follows: diabetes (250), hypertension (401-405), hyperlipidemia (272), coronary artery disease (CAD; 410-414), cerebrovascular disease (430-438), and atrial fibrillation (427.31).

\section{Statistical analysis}

All statistical analyses were performed using SAS version 9.4 (SAS Institute, Cary, NC, USA). The distribution of patient demographics, catastrophic illness certificates, and comorbidities between the four groups were examined using analysis of variance (ANOVA) for the continuous variables, and Pearson's chi-squared test or Fisher's exact test for the categorical variables, where appropriate. The cumulative incidence of dementia by the cDDDs of anticholinergic use was estimated using the Kaplan-Meier method, with comparisons between groups conducted using the log-rank test. We had tested the proportional hazards assumption and the assumption was satisfied [25]. Univariate and multivariable Cox proportional hazard models were then performed to assess the cDDDs of anticholinergic use associated with the risk of subsequent dementia. To check the potential bias of reverse causation, a sensitivity test was performed with multivariable Cox proportional hazard regression analyses by excluding not only 
the patients diagnosed with dementia within 1 year from the index date, but also within two, three or 4 years to investigate the dementia risk among LUTS patients with different cDDDs. The robust sandwich variance estimator was used to reduce the potential bias of standard errors in misspecification of clustering samples and to increase valid statistical inferences about the corresponding covariate effects $[25,26]$. We estimated hazard ratios (HRs) with 95\% confidence intervals (CIs). Statistical significance was set at $p<0.05$ and all tests were two-tailed.

\section{Results}

Characteristics of the participants, and each group categorized by cDDD are shown in Table 1 . At the end of December 2005, 16,412 patients newly diagnosed with LUTS were included; the mean age was 66.5 years, and the majority were male. Of these, by the final follow up, 13,681 patients had consumed less than 28 cDDDs of anticholinergic medications, 1285 patients had taken 28-84 cDDDs, 1024 patients had taken 85-336 cDDDs, and 422 patients had received more than $337 \mathrm{cDDDs}$ of anticholinergics. We also analyzed catastrophic illness certificates and other known comorbidities. Patients with LUTS who consumed more cumulative doses of anticholinergics were more likely to be older and have a higher prevalence of diabetes mellitus.

At the end of the follow-up period, 1666 patients had dementia. The mean (SD) and median (IQR) follow-up time to dementia was 9.0 (2.2) years and $9.2(8.0-10.7)$ years respectively for all patients, and were all longer than 9 years in each cDDDs group. Dementia incidence was $10 \%$ in the $<28$ cDDD group, $8.9 \%$ in the $28-84$ cDDD group, $11.5 \%$ in the $85-336$ cDDD group, and $14.4 \%$ in the more than $337 \mathrm{cDDD}$ group $(p=.005)$. The incidence of dementia was significantly related to higher cumulative doses of anticholinergic use in patients with LUTS. We further analyzed the $<28$ cDDD and $28-84$ cDDD groups, and found no significant difference between these two groups in dementia incidence $(p=.326)$.

In Cox proportional hazards regression analyses (Table 2), the adjusted HR for dementia was 1.15 (95\% CI 0.97-1.37) in the $85-336$ cDDD group and 1.40 (95\% CI 1.12-1.75) in the highest exposure ( $\geq 337$ cDDDs) group after adjusting

Table 1 Characteristics of patients with lower urinary tract symptoms by categories of cumulative defined daily doses of therapeutic bladder anticholinergics

\begin{tabular}{|c|c|c|c|c|c|c|c|c|c|c|c|}
\hline & \multicolumn{2}{|c|}{$\begin{array}{l}\text { Total } \\
(n=16,412) \\
\end{array}$} & \multicolumn{2}{|c|}{$\begin{array}{l}<28 \text { cDDDs } \\
(n=13,681) \\
\end{array}$} & \multicolumn{2}{|c|}{$\begin{array}{l}28-84 \text { cDDDs } \\
(n=1285) \\
\end{array}$} & \multicolumn{2}{|c|}{$\begin{array}{l}85-336 \text { cDDDs } \\
(n=1024)\end{array}$} & \multicolumn{2}{|c|}{$\begin{array}{l}\geq 337 \text { cDDDs } \\
(n=422)\end{array}$} & \multirow[t]{2}{*}{$P$} \\
\hline & $n$ & (\%) & $n$ & (\%) & $\mathrm{n}$ & (\%) & $n$ & (\%) & $\mathrm{n}$ & (\%) & \\
\hline \multicolumn{12}{|l|}{ Baseline characteristics } \\
\hline Age (years), mean (SD) & 66.5 & (9.6) & 66.4 & $(9.8)$ & 67.1 & $(8.8)$ & 67.2 & $(8.5)$ & 67.9 & $(8.1)$ & $<0.001$ \\
\hline $50-59$ & 4346 & $(26.5)$ & 3787 & $(27.7)$ & 277 & $(21.6)$ & 206 & $(20.1)$ & 76 & (18.0) & $<0.001$ \\
\hline $60-69$ & 5421 & $(33.0)$ & 4441 & (32.4) & 447 & $(34.8)$ & 377 & $(36.8)$ & 156 & $(37.0)$ & \\
\hline $70-79$ & 5192 & (31.6) & 4173 & $(30.5)$ & 482 & $(37.5)$ & 377 & $(36.8)$ & 160 & $(37.9)$ & \\
\hline$\geq 80$ & 1453 & $(8.9)$ & 1280 & $(9.4)$ & 79 & $(6.1)$ & 64 & $(6.3)$ & 30 & $(7.1)$ & \\
\hline Sex & & & & & & & & & & & $<0.001$ \\
\hline Male & 13,745 & $(83.7)$ & 11,312 & $(82.7)$ & 1130 & $(87.9)$ & 919 & $(89.7)$ & 384 & $(91.0)$ & \\
\hline Female & 2667 & $(16.3)$ & 2369 & (17.3) & 155 & $(12.1)$ & 105 & $(10.3)$ & 38 & $(9.0)$ & \\
\hline Catastrophic illness certificate & 2496 & $(15.2)$ & 2094 & (15.3) & 181 & $(14.1)$ & 142 & $(13.9)$ & 79 & $(18.7)$ & 0.078 \\
\hline \multicolumn{12}{|l|}{ Comorbidities } \\
\hline Diabetes mellitus & 1093 & $(6.7)$ & 950 & $(6.9)$ & 56 & $(4.5)$ & 57 & $(5.6)$ & 30 & $(7.1)$ & 0.002 \\
\hline Hyperlipidemia & 236 & $(1.4)$ & 199 & $(1.5)$ & 16 & $(1.3)$ & 15 & $(1.5)$ & 6 & $(1.4)$ & 0.946 \\
\hline Hypertension & 2019 & $(12.3)$ & 1706 & $(12.5)$ & 128 & $(10.0)$ & 134 & $(13.1)$ & 51 & $(12.1)$ & 0.058 \\
\hline$C A D$ & 391 & $(2.4)$ & 323 & $(2.4)$ & 32 & $(2.5)$ & 23 & $(2.3)$ & 13 & $(3.1)$ & 0.787 \\
\hline CVD & 452 & $(2.8)$ & 392 & $(2.9)$ & 27 & $(2.1)$ & 23 & $(2.3)$ & 10 & $(2.4)$ & 0.273 \\
\hline Atrial fibrillation & 44 & $(0.3)$ & 37 & $(0.3)$ & 2 & $(0.2)$ & 4 & $(0.4)$ & 1 & $(0.2)$ & 0.753 \\
\hline Depression & 40 & $(0.2)$ & 33 & $(0.2)$ & 0 & $(0.0)$ & 5 & $(0.5)$ & 2 & $(0.5)$ & 0.087 \\
\hline $\mathrm{TB} \mid$ & 20 & $(0.1)$ & 19 & $(0.1)$ & 1 & $(0.1)$ & 0 & $(0.0)$ & 0 & $(0.0)$ & 0.254 \\
\hline \multicolumn{12}{|l|}{ Follow-up characteristics } \\
\hline Number of outpatient visits per year, mean (SD) & 27.4 & $(19.9)$ & 27.1 & (19.8) & 28.0 & $(20.2)$ & 28.6 & $(19.5)$ & 29.8 & (20.2) & 0.003 \\
\hline Dementia & 1666 & $(10.2)$ & 1372 & $(10.0)$ & 115 & (8.9) & 118 & $(11.5)$ & 61 & $(14.4)$ & 0.005 \\
\hline
\end{tabular}


Table 2 Cox proportional hazards regression analyses for the risk of dementia among patients with lower urinary tract symptoms and different cumulative defined daily doses of therapeutic bladder anticholinergics

\begin{tabular}{|c|c|c|c|c|c|c|}
\hline & \multicolumn{3}{|c|}{ Univariate model } & \multicolumn{3}{|c|}{ Multivariate model } \\
\hline & $\mathrm{HR}$ & $(95 \% \mathrm{Cl})$ & $P$ & $\mathrm{HR}$ & $(95 \% \mathrm{Cl})$ & $P$ \\
\hline \multicolumn{7}{|l|}{ cDDD category } \\
\hline$<28$ & 1.00 & & & 1.00 & & \\
\hline $28-84$ & 0.86 & $(0.70-1.05)$ & 0.127 & 0.88 & $(0.73-1.06)$ & 0.178 \\
\hline $85-336$ & 1.11 & $(0.94-1.32)$ & 0.221 & 1.15 & $(0.97-1.37)$ & 0.107 \\
\hline$\geq 337$ & 1.39 & $(1.10-1.76)$ & 0.006 & 1.40 & $(1.12-1.75)$ & 0.004 \\
\hline Age (years) & 1.08 & $(1.08-1.09)$ & $<0.001$ & 1.08 & $(1.08-1.09)$ & $<0.001$ \\
\hline \multicolumn{7}{|l|}{ Sex } \\
\hline Male & 1.00 & & & 1.00 & & \\
\hline Female & 1.16 & $(1.02-1.31)$ & 0.023 & 1.35 & $(1.19-1.53)$ & $<0.001$ \\
\hline Catastrophic illness certificate & 1.54 & $(1.35-1.76)$ & $<0.001$ & 1.24 & $(1.09-1.42)$ & 0.001 \\
\hline \multicolumn{7}{|l|}{ Comorbidities } \\
\hline Diabetes mellitus & 1.23 & $(1.04-1.46)$ & 0.018 & 1.15 & $(0.96-1.37)$ & 0.121 \\
\hline Hyperlipidemia & 1.24 & $(0.84-1.83)$ & 0.274 & 1.53 & $(1.04-2.24)$ & 0.029 \\
\hline Hypertension & 1.20 & $(1.03-1.41)$ & 0.023 & 0.96 & $(0.82-1.13)$ & 0.659 \\
\hline CAD & 0.99 & $(0.72-1.36)$ & 0.963 & 0.72 & $(0.53-0.97)$ & 0.034 \\
\hline CVD & 2.46 & $(1.89-3.21)$ & $<0.001$ & 1.92 & $(1.46-2.52)$ & $<0.001$ \\
\hline Atrial fibrillation & 2.21 & $(1.18-4.12)$ & 0.013 & 1.41 & $(0.75-2.63)$ & 0.281 \\
\hline Number of outpatient visits per year & 1.01 & $(1.01-1.02)$ & $<0.001$ & 1.01 & $(1.00-1.01)$ & $<0.001$ \\
\hline
\end{tabular}

$C A D$ Coronary artery disease, $C D D D$ s Cumulative defined daily doses, $C I$ Confidence interval, $C V D$ Cerebrovascular disease, $H R$ Hazard ratio.

for age, sex, catastrophic illness certificates and comorbidities of interest. The sensitivity test of the risk of dementia after excluding those who were diagnosed within 2 , 3, or 4 years among patients with LUTS prescribed with different cDDDs is shown in Table 3. We found a trend for adjusted HRs, which increased for the groups with higher cDDDs, when the patients with LUTS who were diagnosed with dementia within 2 , 3, or 4 years had been sequentially excluded. In our analysis, higher cumulative doses of anticholinergics manifested as a strong risk factor for dementia in patients with LUTS.

The Kaplan-Meier plot (Fig. 2) with the time / number of dementia cases from the index date showed that there was a significant difference in the incidence of dementia between the four groups of patients with LUTS when classified by cumulative anticholinergic doses (logrank test $p=.015)$. The highest cumulative exposure group, which consumed $\geq 337$ cDDDs, had the highest risk of dementia.

\section{Discussion}

In this nationwide population-based cohort study using data from Taiwan's NHIRD, we found a significant correlation between cumulative exposure to anticholinergics and the incidence of dementia in patients with LUTS. The HR for dementia in those who consumed $\geq 337$

Table 3 Multivariate Cox proportional hazards regression analyses for the risk of dementia after excluding those who were diagnosed within 2, 3, or 4 years among patients with lower urinary tract symptom prescribed with different cumulative defined daily doses of therapeutic bladder anticholinergics

\begin{tabular}{|c|c|c|c|c|c|c|c|c|c|c|c|c|}
\hline & \multicolumn{4}{|c|}{ Excluding diagnosis within two years ${ }^{a}$} & \multicolumn{4}{|c|}{ Excluding diagnosis within three years ${ }^{a}$} & \multicolumn{4}{|c|}{ Excluding diagnosis within four years ${ }^{a}$} \\
\hline & Events (\%) & $\mathrm{HR}$ & $(95 \% \mathrm{Cl})$ & $P$ & Events (\%) & $\mathrm{HR}$ & $(95 \% \mathrm{Cl})$ & $P$ & Events (\%) & $\mathrm{HR}$ & $(95 \% \mathrm{Cl})$ & $P$ \\
\hline \multicolumn{13}{|c|}{ cDDD category } \\
\hline$<28$ & $1017(7.4)$ & 1.00 & & & $861(6.3)$ & 1.00 & & & $721(5.3)$ & 1.00 & & \\
\hline $28-84$ & $98(7.6)$ & 1.00 & $(0.82-1.22)$ & 0.995 & $92(7.2)$ & 1.06 & $(0.86-1.31)$ & 0.568 & $83(6.5)$ & 1.15 & $(0.92-1.43)$ & 0.223 \\
\hline $85-336$ & $100(9.8)$ & 1.30 & $(1.07-1.59)$ & 0.009 & $90(8.8)$ & 1.33 & $(1.07-1.65)$ & 0.009 & $77(7.5)$ & 1.36 & $(1.08-1.71)$ & 0.008 \\
\hline$\geq 337$ & $54(12.8)$ & 1.69 & $(1.33-2.15)$ & $<0.001$ & $50(11.8)$ & 1.77 & $(1.39-2.26)$ & $<0.001$ & $44(10.4)$ & 1.88 & $(1.44-2.46)$ & $<0.001$ \\
\hline
\end{tabular}

${ }^{a}$ Adjusted for patient's age, sex, catastrophic illness certificates, comorbidities, and number of outpatient visits $C D D D$ s Cumulative defined daily doses, $\mathrm{Cl}$ Confidence interval, HR Hazard ratio. 


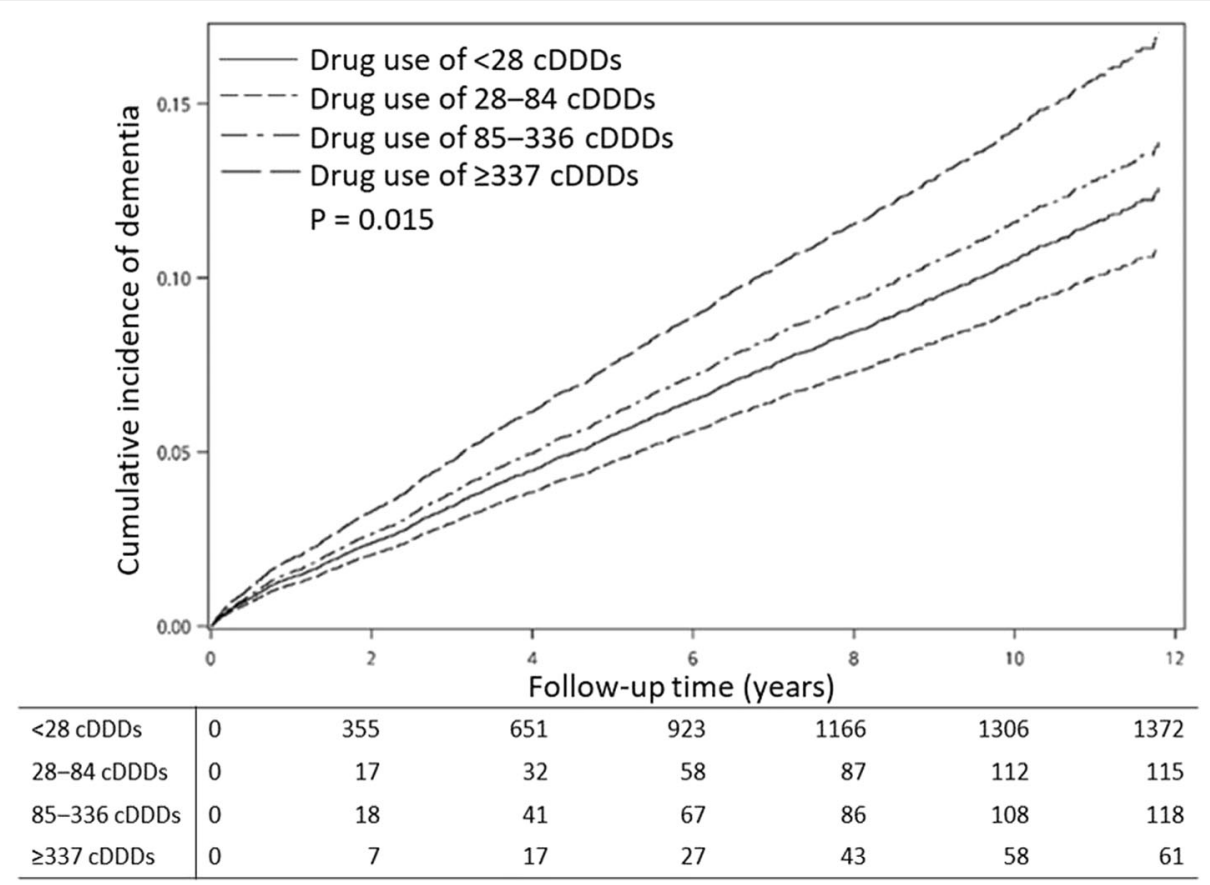

Fig. 2 The cumulative incidence of dementia in patients with lower urinary tract symptoms by cumulative defined daily doses of therapeutic bladder anticholinergics

cDDDs was $1.40(95 \%$ CI 1.12-1.75) compared with those who consumed $<28$ cDDDs. To our knowledge, few studies have focused on the dose effect of bladder anticholinergics on the risk of dementia in Asia.

A systematic review analyzing 46 studies found that the use of anticholinergic agents significantly increased the risk of cognitive decline [27]. A large prospective cohort study revealed that higher cumulative use of strong anticholinergics in patients older than 65 years was associated with an increased risk of dementia, and the effect appeared to be lifelong [22].

A recent nested case-control study from general practices in England also concluded that exposure to higher total cumulative doses of anticholinergics was associated with increased risk of dementia in patients older than 55 years, especially in those who consumed more anticholinergic antidepressants, antiparkinson drugs, antipsychotics, bladder antimuscarinic drugs and antiepileptics [28]. Elderly patients using anticholinergics for overactive bladder have been found to have a higher incidence of dementia [29], especially when the anticholinergics have a small molecular size, a neutral charge, are lipophilic, are hydrophobic, and can cross the blood brain barrier easily [30]. The association between LUTS and increased dementia risk was demonstrated in our previous study [17], but the potential contributing factors remain unclear. Bladder anticholinergics are used very widely in the standardized therapy for LUTS, however, little is known about whether this increases the risk of dementia in this patient group or not. We aimed to clinically produce evidence for physicians to weigh-up the benefits and risks of bladder anticholinergics in LUTS treatment, especially in an Asian population. Therefore, we further focused our study on the use of therapeutic bladder anticholinergics only in patients with LUTS in Taiwan. Our findings regarding the cumulative dose effect of bladder anticholinergics echoes the study by Coupland et al. [28] and highlights that this should be considered in clinical practice.

Two randomized controlled trials published in 2005 [31] and 2017 [32] revealed no cognitive decline among elderly patients with LUTS who used Darifenacin or Solifenacin, respectively. These results could be explained by differential selectivity to muscarinic receptors. Among muscarinic receptors, M1-M2 receptors are located in the brain and are important for cognition management, while the M2 and M3 receptors are located in the bladder [30, 33]. If anticholinergics do not selectively block muscarinic receptors, this could increase the risk of cognitive decline. In the two studies above, Darifenacin and Solifenacin are both M3receptor-selective anticholinergics; therefore, there was no observation of cognitive decline [31, 32]. This difference could be because we focused on the cumulative dose effect of anticholinergic exposure instead of the various properties of each anticholinergic agent. Differences in the selectivity of the M3 muscarinic receptor between each anticholinergic agent should be assessed in future studies. 
Conventionally, anticholinergic-induced cognitive impairment appears reversible once the medication has been discontinued [34]. However, there are increasing concerns that the effect might be lifelong [22, 35-37]. A biologically plausible mechanism could be that the cumulative use of these drugs can result in pathological changes similar to those occurring in Alzheimer's disease [38]. A recent study evaluating structural and functional brain changes in patients who use anticholinergics via structural magnetic resonance imaging, fluorodeoxyglucose $\mathrm{F} 18$ positron emission tomography, and cognitive testing concluded that anticholinergic agents increase whole brain atrophy, reduce glucose metabolism, and cause further clinical decline [21]. Anticholinergics that alter cholinergic pathways are associated with an increased risk of brain atrophy and a decrease in brain function. Reduced cholinergic activity caused by anticholinergics can lead to cell death and neurodegeneration [39-41]. Another hypothesis is that anticholinergics might increase serum corticosterone levels via regulating the hypothalamic-pituitary-adrenal axis, thus promoting neuronal damage in response to stress [42].

Our study has some strengths. First, it was a nationwide cohort study with an adequate follow-up period and a very low attrition rate. Second, although the results of most studies focusing on the long-term use of a single anticholinergic agent have found an increased risk of developing dementia, our results demonstrate that either using one anticholinergic agent or switching anticholinergic agents can cumulatively increase the risk of dementia. Third, although most studies have focused on elderly patients over 65 years, our study included patients starting at 50 years of age, and the increased risk of dementia remained.

Our study does have some limitations. First, there is no gold standard for anticholinergic burden measurement. We focused on the cumulative dose effect of anticholinergic use with incident dementia risk. Second, in this database study, measurement of the exposure relies on the prescriptions filled, and this may not reflect the true amount of medication taken by the patient. Third, common anticholinergics with an ACB score of 3 (definite anticholinergic activity) associated with an increased risk of dementia also include antidepressant, urological and antiparkinson medications [23]. However, we only calculated cumulative doses of bladder anticholinergics available in Taiwan because we wanted to clarify the association of bladder anticholinergics and dementia risk in LUTS patients to inform benefits and risks in clinical practice. Fifth, since LUTS and dementia are both multifactorial disorders, they may share common pathophysiological pathways, that is, LUTS may be symptoms of dementia. Therefore, there is a risk of reverse causation. To explore this, we performed multivariable Cox proportional hazard regression analyses as a sensitivity test, by excluding dementia diagnosis not only within 1 year from index date, but also within 2, 3 or 4 years. We used Cox proportional hazards regression analyses to adjust for important comorbidities and possible confounding factors as far as possible [43]. However, as with many pharmacoepidemiologic studies, we cannot fully account for residual confounding and bias, and factors such as variable prescribing patterns, comorbidities, prognosis and controlling for the time dependency of drug use.

\section{Conclusions}

In this study, we have shown that high cumulative exposure of therapeutic bladder anticholinergics can increase the risk of dementia in people with LUTS. Therefore, anticholinergics should be used with caution in patients with LUTS. Physicians should review and weigh the need for anticholinergics in clinical practice. Behavioral therapy or other medications such as beta-3-agonists could be considered as alternative options. Future work should focus on the properties of various anticholinergic agents, to discover whether they have a differential impact on cognitive function.

\section{Abbreviations}

ACB: Anticholinergic Cognitive Burden; cDDDs: Cumulative defined daily doses; LHID: Longitudinal Health Insurance Database; LUTS: Lower urinary tract symptoms; NHIRD: National Health Insurance Research Database

\section{Acknowledgements \\ This paper uses data from Taiwan National Health Insurance Research Database. This database is supported by the National Health Research Institutes (NHRI). The authors thank all the patients who included in the study. We further acknowledge the suggestions and proofreading by Prof. Elizabeth L. Sampson at University College London, and the help from Prof. Hung-Pin Tu and Prof. Huey-Juan Lin.}

\section{Authors' contributions}

YC-Conceptualization, validation, formal analysis, investigation, data curation, original draft preparation, project administration. YL- Conceptualization, validation, formal analysis, investigation, data curation, original draft preparation, project administration. CC- Conceptualization, methodology, software, validation, formal analysis, investigation, resources, data curation, original draft preparation, review and editing, supervision. MJ-

Conceptualization, validation, formal analysis, data curation, original draft preparation. $\mathrm{CH}$ - validation, formal analysis, data curation. MP- software, validation, formal analysis, resources, data curation, supervision. YTmethodology, software, validation, formal analysis, data curation. $\mathrm{CH}$ validation, formal analysis, data curation. PJ- Conceptualization, methodology, validation, formal analysis, investigation, data curation, original draft preparation, review and editing, supervision, project administration, funding acquisition. All authors read and approved the final manuscript.

\section{Funding}

This research was funded by the Kaohsiung Medical University Hospital (Grant reference: KMUH106-6R74). The funders had no role in study design, data collection and analysis, decision to publish, or preparation of the manuscript.

\section{Availability of data and materials}

The data that support the findings of this study are available from Taiwan National Health Insurance Research Database but restrictions apply to the availability of these data, which were used under license for the current study, and so are not publicly available. Data are however available from the authors upon reasonable request and with permission of Taiwan National Health Insurance Research Database. 


\section{Ethics approval and consent to participate}

Ethics permission was approved by the Institutional Review Board of Chi Mei Medical Center (IRB No. 10708-E01), and the requirement for informed consent was waived. The data for this study was obtained from the National Health Insurance Research Database (NHIRD, http://nhird.nhri.org.tw/). The application of permission to access the data was sent to National Health Research Institute (NHRI), Taiwan and approved. The NHIRD has been transferred to the Health and Welfare Data Science Center (HWDC), Department of Statistics, Ministry of Health and Welfare, Taiwan (http://dep. mohw.gov.tw/DOS/np-2497-113.html).

\section{Consent for publication}

Not applicable.

\section{Competing interests}

The authors declare that they have no competing interests.

\section{Author details}

'Department of Family Medicine, Kaohsiung Municipal Hsiao-Kang Hospital, Kaohsiung Medical University, Kaohsiung City 812, Taiwan. ${ }^{2}$ Department of Family Medicine, Kaohsiung Municipal Ta-Tung Hospital, Kaohsiung Medical University, Kaohsiung City 801, Taiwan. ${ }^{3}$ Department of Healthcare Administration, College of Medicine, I-Shou University, Kaohsiung City 824, Taiwan. ${ }^{4}$ Department of Medical Research, Chi-Mei Medical Center, Tainan City 710, Taiwan. ${ }^{5}$ Department of Hospital and Health Care Administration, Chia Nan University of Pharmacy and Science, Tainan City 717, Taiwan. ${ }^{6}$ Center for Big Data Analytics and Statics, Chang Gung Memorial Hospital, Taoyuan City 333, Taiwan. 'Division of Urogynecology, Department of Obstetrics and Gynecology, Chi Mei Foundation Hospital, Tainan, Taiwan. ${ }^{8}$ Department of Obstetrics and Gynecology, College of Medicine, Fu-Jan Catholic University, New Taipei City, Taiwan. ${ }^{9}$ Department of Family Medicine, Kaohsiung Medical University Hospital, Kaohsiung Medical University, Kaohsiung City 807, Taiwan. ${ }^{10}$ School of Medicine, College of Medicine, Kaohsiung Medical University, Kaohsiung City 807, Taiwan. ${ }^{11}$ Marie Curie Palliative Care Research Department, Division of Psychiatry, University College London, London W1T 7NF, UK. ${ }^{12}$ Palliative Care Center, Chi-Mei Medical Center, Tainan City 710, Taiwan.

Received: 23 January 2019 Accepted: 22 December 2019 Published online: 30 December 2019

\section{References}

1. Abrams P, Chapple C, Khoury S, Roehrborn C, de la Rosette J. Evaluation and treatment of lower urinary tract symptoms in older men. J Urol. 2009; 181(4):1779-87.

2. Taylor BC, Wilt TJ, Fink HA, Lambert LC, Marshall LM, Hoffman AR, Beer TM, Bauer DC, Zmuda JM, Orwoll ES. Prevalence, severity, and health correlates of lower urinary tract symptoms among older men: the MrOS study. Urology. 2006:68(4):804-9.

3. Parsons JK, Bergstrom J, Silberstein J, Barrett-Connor E. Prevalence and characteristics of lower urinary tract symptoms in men aged $>$ or $=80$ years. Urology. 2008;72(2):318-21.

4. Miu DK, Lau S, Szeto SS. Etiology and predictors of urinary incontinence and its effect on quality of life. Geriatr Gerontol Int. 2010;10(2):177-82.

5. Helfand BT, McVary KT, Meleth S, Sharp V, Foster H, Naslund M, Williams OD The relationship between lower urinary tract symptom severity and sleep disturbance in the CAMUS trial. J Urol. 2011;185(6):2223-8.

6. Coyne KS, Wein AJ, Tubaro A, Sexton CC, Thompson CL, Kopp ZS, Aiyer LP. The burden of lower urinary tract symptoms: evaluating the effect of LUTS on health-related quality of life, anxiety and depression: EpiLUTS. BJU Int. 2009;103(Suppl 3):4-11.

7. Laumann EO, Kang JH, Glasser DB, Rosen RC, Carson CC. Lower urinary tract symptoms are associated with depressive symptoms in white, black and Hispanic men in the United States. J Urol. 2008;180(1):233-40.

8. Talley KM, Wyman JF, Bronas UG, Olson-Kellogg BJ, McCarthy TC, Zhao H. Factors associated with toileting disability in older adults without dementia living in residential care facilities. Nurs Res. 2014;63(2):94-104.

9. Lawhorne LW, Ouslander JG, Parmelee PA, Resnick B, Calabrese B. Urinary incontinence: a neglected geriatric syndrome in nursing facilities. J Am Med Dir Assoc. 2008;9(1):29-35.
10. Wagg A, Gibson W, Ostaszkiewicz J, Johnson T 3rd, Markland A, Palmer MH, Kuchel G, Szonyi G, Kirschner-Hermanns R. Urinary incontinence in frail elderly persons: report from the 5 th international consultation on incontinence. NeurourolUrodyn. 2015;34(5):398-406.

11. Braak H, Braak E. Frequency of stages of Alzheimer-related lesions in different age categories. Neurobiol Aging. 1997;18(4):351-7.

12. Wolters FJ, Ikram MA. Epidemiology of dementia: the burden on society, the challenges for research. Methods Mol Biol (Clifton, NJ). 2018;1750:3-14.

13. Heinen I, van den Bussche H, Koller D, Wiese B, Hansen H, Schafer I, Scherer $\mathrm{M}$, Schon G. Kaduszkiewicz H: [morbidity differences according to nursing stage and nursing setting in long-term care patients: results of a claims data based study]. Z Gerontol Geriatr. 2015;48(3):237-45.

14. Kamiya M, Sakurai T, Ogama N, Maki Y, Toba K. Factors associated with increased caregivers' burden in several cognitive stages of Alzheimer's disease. Geriatr Gerontol Int. 2014;14(Suppl 2):45-55.

15. LoGiudice D, Watson R. Dementia in older people: an update. Intern Med J. 2014;44(11):1066-73.

16. Livingston G, Sommerlad A, Orgeta V, Costafreda SG, Huntley J, Ames D, Ballard C, Banerjee S, Burns A, Cohen-Mansfield J, et al. Dementia prevention, intervention, and care. Lancet (London, England). 2017; 390(10113):2673-734.

17. Chiang CH, Wu MP, Ho CH, Weng SF, Huang CC, Hsieh WT, Hsu YW, Chen PJ. Lower urinary tract symptoms are associated with increased risk of dementia among the elderly: a Nationwide study. Biomed Res Int. 2015;2015:187819.

18. Sakakibara R, Panicker J, Fowler CJ, Tateno F, Kishi M, Tsuyusaki Y, Yamanishi T, Uchiyama T, Yamamoto T, Yano M. Is overactive bladder a brain disease? The pathophysiological role of cerebral white matter in the elderly. Int J Urol. 2014;21(1):33-8.

19. Kevin T, McVary M. FACSRajiv Saini, MD: Lower urinary tract symptoms in men. In: Post TW, editor. UpToDate. Waltham: UpToDate; 2018.

20. Nabi G, Cody JD, Ellis G, Hay-Smith J, Herbison GP. Anticholinergic drugs versus placebo for overactive bladder syndrome in adults. Cochrane Database Syst Rev. 2006;(4). Art. No.: CD003781. https://doi.org/10.1002/ 14651858.CD003781.pub2.

21. Risacher SL, McDonald BC, Tallman EF, West JD, Farlow MR, Unverzagt FW, Gao S, Boustani M, Crane PK, Petersen RC, et al. Association between anticholinergic medication use and cognition, brain metabolism, and brain atrophy in cognitively normal older adults. JAMA Neurol. 2016;73(6):721-32.

22. Gray SL, Anderson ML, Dublin S, Hanlon JT, Hubbard R, Walker R, Yu O, Crane PK, Larson EB. Cumulative use of strong anticholinergics and incident dementia: a prospective cohort study. JAMA Intern Med. 2015;175(3):401-7.

23. Richardson K, Fox C, Maidment I, Steel N, Loke YK, Arthur A, Myint PK, Grossi CM, Mattishent K, Bennett K, et al. Anticholinergic drugs and risk of dementia: case-control study. BMJ (Clinical research ed). 2018;361:k1315.

24. Bentkover J, Cai S, Makineni R, Mucha L, Treglia M, Mor V. Road to the nursing home: costs and disease progression among medicare beneficiaries with ADRD. Am J Alzheimers Dis Other Demen. 2012;27(2):90-9.

25. Lin DY, Wei LJ. The robust inference for the Cox proportional hazards model. J Am Stat Assoc. 1989;84(408):1074-8.

26. Desai M, Bryson SW, Robinson T. On the use of robust estimators for standard errors in the presence of clustering when clustering membership is misspecified. Contemp Clin Trials. 2013;34(2):248-56.

27. Fox C, Smith T, Maidment I, Chan WY, Bua N, Myint PK, Boustani M, Kwok CS, Glover M, Koopmans I, et al. Effect of medications with anti-cholinergic properties on cognitive function, delirium, physical function and mortality: a systematic review. Age Ageing. 2014;43(5):604-15.

28. Coupland CAC, Hill T, Dening T, Morriss R, Moore M, Hippisley-Cox J. Anticholinergic drug exposure and the risk of dementia: a nested Ccasecontrol study. JAMA Intern Med. 2019;179(8):1084-93.

29. Araklitis G, Cardozo L. Safety issues associated with using medication to treat overactive bladder. Expert Opin Drug Saf. 2017;16(11):1273-80.

30. Chancellor MB, Staskin DR, Kay GG, Sandage BW, Oefelein MG, Tsao JW. Blood-brain barrier permeation and efflux exclusion of anticholinergics used in the treatment of overactive bladder. Drugs Aging. 2012;29(4):259-73.

31. Lipton RB, Kolodner K, Wesnes K. Assessment of cognitive function of the elderly population: effects of darifenacin. J Urol. 2005;173(2):493-8.

32. Kosilov K, Kuzina I, Kuznetsov V, Gainullina Y, Kosilova L, Prokofyeva A, Loparev S. Cognitive functions and health-related quality of life in men with benign prostatic hyperplasia and symptoms of overactive bladder when treated with a combination of tamsulosin and solifenacin in a higher dosage. Aging Male. 2018;21(2):121-9. 
33. Kay GG, Granville LJ. Antimuscarinic agents: implications and concerns in the management of overactive bladder in the elderly. Clin Ther. 2005;27(1): 127-38 quiz 139-140.

34. Tannenbaum C, Paquette A, Hilmer S, Holroyd-Leduc J, Carnahan R. A systematic review of amnestic and non-amnestic mild cognitive impairment induced by anticholinergic, antihistamine, GABAergic and opioid drugs. Drugs Aging. 2012;29(8):639-58

35. Jessen F, Kaduszkiewicz H, Daerr M, Bickel H, Pentzek M, Riedel-Heller S, Wagner M, Weyerer S, Wiese B, van den Bussche $\mathrm{H}$, et al. Anticholinergic drug use and risk for dementia: target for dementia prevention. Eur Arch Psychiatry Clin Neurosci. 2010;260(Suppl 2):S111-5.

36. Ancelin ML, Artero S, Portet F, Dupuy AM, Touchon J, Ritchie K. Nondegenerative mild cognitive impairment in elderly people and use of anticholinergic drugs: longitudinal cohort study. BMJ (Clinical research ed). 2006:332(7539):455-9.

37. Carriere I, Fourrier-Reglat A, Dartigues JF, Rouaud O, Pasquier F, Ritchie K, Ancelin ML. Drugs with anticholinergic properties, cognitive decline, and dementia in an elderly general population: the 3-city study. Arch Intern Med. 2009;169(14):1317-24.

38. Perry EK, Kilford L, Lees AJ, Burn DJ, Perry RH. Increased Alzheimer pathology in Parkinson's disease related to antimuscarinic drugs. Ann Neurol. 2003;54(2):235-8.

39. Everitt BJ, Robbins TW. Central cholinergic systems and cognition. Annu Rev Psychol. 1997:48:649-84.

40. Del Pino J, Zeballos G, Anadon MJ, Moyano P, Diaz MJ, Garcia JM, Frejo MT. Cadmium-induced cell death of basal forebrain cholinergic neurons mediated by muscarinic M1 receptor blockade, increase in GSK-3beta enzyme, beta-amyloid and tau protein levels. Arch Toxicol. 2016;90(5):1081-92.

41. Geula C. Abnormalities of neural circuitry in Alzheimer's disease: hippocampus and cortical cholinergic innervation. Neurology. 1998:51(1 Suppl 1):S18-29 discussion S65-17.

42. Paul S, Jeon WK, Bizon JL, Han JS. Interaction of basal forebrain cholinergic neurons with the glucocorticoid system in stress regulation and cognitive impairment. Front Aging Neurosci. 2015:7:43.

43. Gill SS, Bai AD. Beta testing the potential link between the alpha antagonist tamsulosin and dementia. Pharmacoepidemiol Drug Saf. 2018;27(3):349-50.

\section{Publisher's Note}

Springer Nature remains neutral with regard to jurisdictional claims in published maps and institutional affiliations.

Ready to submit your research? Choose BMC and benefit from:

- fast, convenient online submission

- thorough peer review by experienced researchers in your field

- rapid publication on acceptance

- support for research data, including large and complex data types

- gold Open Access which fosters wider collaboration and increased citations

- maximum visibility for your research: over $100 \mathrm{M}$ website views per year

At $\mathrm{BMC}$, research is always in progress.

Learn more biomedcentral.com/submissions 